\title{
O ensino de custos nas escolas de graduação em enfermagem*
}

\author{
COST TEACHING IN GRADUATE NURSING SCHOOLS
}

LA ENSEÑANZA DE COSTOS EN LAS ESCUELAS DE PRE GRADO EN ENFERMERÍA

Ivone Maria Fonseca Francisco', Valéria Castilho²

\section{RESUMO}

Trata-se de um estudo que teve como objetivo principal analisar a inserção de conteúdos relativos a Custos na disciplina Administração Aplicada à Enfermagem das Escolas de Graduação em Enfermagem do Estado de São Paulo. A amostra foi composta por 28 docentes. $O$ estudo foi do tipo descritivo, exploratório, correlacional com abordagem quantitativa. Os resultados permitiram constatar que 19 (67,86\%) docentes ministram esse tema na graduação, não havendo correlação ou associação estatisticamente significativa entre o ensino do Tema Custos, o número de pósgraduções do docente e o tipo de instituição em que o docente trabalha (Pública ou Privada).

\section{PALAVRAS-CHAVE}

Enfermagem.

Controle de Custos.

Ensino superior.

Ensino

\begin{abstract}
The present study intends to analyse the implement of content related to Costs in the Management Applied to Nursing subject in Graduate Nursing Schools in Sao Paulo State. The descriptive, exploratory associative study with a quantitative approach was put into effect in 28 professionals at the Nursing Schools. The results demonstrated that 19 $(67,86 \%)$ professors teach that subject in graduation. No statistically significant correlation or association has been found among Cost Teaching, the Number of Post Graduation staff and the type of Institutions they work for (Public or Private).
\end{abstract}

\section{KEYWORDS}

Nursing.

Cost control.

Higher education.

Teaching.

\section{RESUMEN}

Este estudio tuvo como objetivo principal analizar la inclusión de contenidos relativos a costos en la disciplina Administración Aplicada a la Enfermería en las Escuelas de Pre Grado en Enfermería del Estado de São Paulo. La muestra estuvo compuesta por 28 docentes. El estudio fue de tipo descriptivo, exploratorio, correlacional con abordaje cuantitativo. Los resultados permitieron constatar que $19(67,86 \%)$ docentes enseñan este tema en el pre grado, no habiendo relación o asociación estadísticamente significativa entre la enseñanza del tema costos, el número de postgraduados docentes y el tipo de instituciones en la que trabaja el docente (Pública o Privada).

\section{PALABRAS CLAVE}

Enfermería.

Control de costos.

Educación superior.

Enseñanza

\footnotetext{
* Trata-se de parte da dissertação "O ensino de custos nas escolas de graduação em enfermagem" apresentada à Escola de Enfermagem da USP em 2002.

1 Enfermeira. Mestre em Enfermagem pela EEUSP. Docente da Universidade de Mogi das Cruzes.

jaymeffj@terra.com.br 2 Enfermeira. Professora Doutora do Departamento de Orientação Profissional da EEUSP. valeriac@usp.br
} 


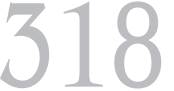

Rev Esc Enferm USP 2004; 38(3): 317-25.

\section{INTRODUÇÃO}

O estudo de aspectos econômicos em saúde mostra-se extremamente atual e oportuno, uma vez que as organizações de saúde vivem momentos de grande competitividade, de busca pela qualidade do atendimento aos clientes e de necessidade de incorporação de tecnologia de ponta.

De acordo com o Conselho Internacional de Enfermagem ${ }^{(1)}(\mathrm{ICN})$, todos os países compartilham de um problema comum, de custos galopantes na área de saúde frente a recursos ou orçamentos limitados.

Em decorrência do significativo aumento de competitividade, as organizações têm como alternativas, maximizar as receitas, pela quantidade e diversificação dos serviços prestados, ou minimizar as despesas, reduzindo continuamente seus custos através da padronização de procedimentos médico-hospitalares e ganhos de qualidade e produtividade ${ }^{(2)}$.

Alguns autores ${ }^{(3)}$ afirmam que custos crescentes e recursos escassos têm afetado todos os prestadores de serviços de saúde, e que não houve outro momento, em que as organizações de saúde tiveram de operar com mais eficiência ou ter mais cuidado com as restrições de custos, como na atualidade. Os mesmos $^{(3)}$ comentam que a restrição de custos é responsabilidade de qualquer provedor de assistência à saúde e a sobrevivência de muitas organizações depende de sua capacidade de geri-los.

A necessidade de adoção de medidas racionalizadoras relativas ao funcionamento das estruturas de saúde, frente aos limitados recursos econômicos disponíveis para atendêlas ${ }^{(4)}$, é uma questão também apontada.

Vários autores ${ }^{(5-6)}$ afirmam que o crescimento exponencial dos custos em saúde está diretamente relacionado a uma série de fatores, tais como: o emprego de novas tecnologias; o aumento da expectativa de vida da população; o crescimento da demanda por atendimento, devido a universalização do acesso à saúde; a escassez de mão de obra qualificada, acarretando baixa produtividade; a má gestão das organizações, devido à incapacidade administrativa dos profissionais de saúde; a não implantação de sistemas de controle de custos; e os desperdícios na cadeia produtiva, entre outros.
Frente a esse quadro complexo descrito, o profissional de Enfermagem precisa estar preparado para responder aos novos desafios gerenciais com os quais vai se deparar em sua atividade profissional, entre os quais o gerenciamento de Custos dos Serviços de Saúde, contribuindo para a viabilidade de atendimentos à saúde, tanto no setor Público como no Privado.

Anselmi ${ }^{(4)}$ referindo-se aos enfermeiros afirma que

no momento atual em que o setor saúde passa por reformas estruturais e conjunturais significativas percebe-se fortes exigências à profissão para que assuma um papel efetivo na gestão econômica das instituições de saúde.

A Organização Mundial de Saúde aponta a enfermeira como o profissional da área de saúde com o maior potencial para assegurar uma assistência rentável, ou seja, eficaz em função dos $\operatorname{custos}^{(1)}$.

Isso vem ao encontro com o que alguns estudiosos das tendências mundiais afirmaram em 1993, que as enfermeiras podem ser responsáveis por 40 a $50 \%$ do faturamento dos hospitais ${ }^{(7)}$.

As enfermeiras constituem um nível decisório importante na alocação de recursos, quando determinam em suas unidades de trabalho as prioridades de seus serviços, decidem quem prestará e quanto tempo será despendido nos cuidados, e quais recursos serão empregados ${ }^{(1)}$.

Atualmente, as enfermeiras administradoras estão cada vez mais sendo envolvidas em decisões financeiras e no planejamento orçamentário de suas instituições, tendo que gerir recursos (humanos, materiais e financeiros) muitas vezes escassos ${ }^{(1)}$.

Em relação à preocupação com o custo do atendimento à saúde, observa-se que os aspectos econômico-financeiros relativos à assistência de Enfermagem foram historicamente ignorados, apesar dos enfermeiros efetivamente gerenciarem as unidades assistenciais, através do planejamento, coordenação, supervisão e controle do trabalho, viabilizando, favorecendo e criando condições técnicas e políticas para a realização do mesmo $^{(8)}$. 
Almeida ${ }^{(9)} \mathrm{em} 1984$ apontava para a pequena quantidade de trabalhos publicados sobre custos da assistência de Enfermagem na literatura brasileira, acrescentando que esta matéria nem sequer era objeto de debate ou estudo nos currículos dos cursos de administração, mostrando não haver nos enfermeiros, ainda uma consciência de custos hospitalares e da contribuição da sua equipe para redução ou adequação dos mesmos à eficácia do serviço.

Comenta-se que a educação econômica em saúde deve ser iniciada na escola de Enfermagem e que os instrutores e empregadores devem proporcionar aos enfermeiros os meio de adquirir responsabilidade fiscal, incluindo o planejamento orçamentário nos currículos de Enfermagem e em programas de administração ${ }^{(3)}$.

O profissional enfermeiro, engajado no processo gerencial das Instituições de Saúde, seja como Gerentes ou Diretores de Divisão, de Serviço, ou Chefes de Unidades, necessitam mais do que nunca buscar conhecimentos sobre custos, reconhecendo seu papel como agente de mudanças no alcance de resultados positivos, bem como buscando o equilíbrio entre qualidade, quantidade e recursos limitados.

Entende-se que o Gerenciamento de Custos na Enfermagem é um processo administrativo que visa a tomada de decisão dos enfermeiros em relação a uma eficiente racionalização na alocação de recursos disponíveis e limitados, com o objetivo de alcançar resultados coerentes às necessidades de saúde da clientela e às necessidades/finalidades institucionais. Para tanto, se faz necessário a compreensão de um conjunto de princípios e conhecimentos de análise econômica que viabilizem a escolha de decisões mais convenientes.

A importância do tema em questão levou à indagação sobre como os graduandos de Enfermagem do Estado de São Paulo estariam sendo preparados (capacitados) em relação ao tema Custos, preparação esta necessária à convivência com uma nova realidade de escassez de recursos, restrições financeiras e dificuldades econômicas das organizações de saúde, tanto no setor público como no privado.

Esta pesquisa tem como objetivos caracterizar os docentes que ministram aulas teóri- cas na disciplina Administração Aplicada à Enfermagem, dos Cursos de Graduação em Enfermagem do Estado de São Paulo; analisar a disciplina supra mencionada quanto à inserção de conteúdos relativos a Custos; correlacionar e associar as variáveis ligadas aos docentes com as variáveis ligadas à inserção de Custos na disciplina supra mencionada.

\section{METODOLOGIA}

\section{Tipo de Estudo}

O estudo realizado foi do tipo descritivo, exploratório, transversal, correlacional, comparativo com abordagem quantitativa.

\section{População Alvo}

Foi realizado um levantamento por meio de dados fornecidos pelo COREN-SP e pela SOBRAGEN (Sociedade Brasileira de Gerenciamento em Enfermagem) no mês de Junho de 2001, onde identificou-se 50 Instituições de Ensino Superior (IES) de Graduação em Enfermagem no Estado de São Paulo, sendo essa a base da amostra de conveniência da população alvo. Tal amostra obedeceu ao seguinte critério de elegibilidade:

- Até o início da coleta de dados (outubro de 2001) a Instituição deveria ter pelo menos uma turma de enfermeiros formados.

Foram excluídas 16 Instituições do total de 50 IES de Graduação em Enfermagem, por não estarem dentro do critério estabelecido.

Restaram portanto 34 IES de Graduação em Enfermagem no Estado de São Paulo, sendo 09 (nove) públicas e 25 privadas.

\section{Procedimento de Coleta de Dados}

Para cada uma das 34 IES de Graduação em Enfermagem do Estado de São Paulo, que constituíram a população alvo, foi enviado um questionário via SEDEX, contendo uma carta de apresentação para o representante da Instituição, juntamente com o questionário e com um ofício destinado a um dos docente da Disciplina de Administração Aplicada à Enfermagem, esclarecendo os objetivos e procedimentos que foram utilizados na pesquisa, além do Termo de Consentimento Livre e Esclarecido, e de um envelope previamente selado e preenchido com o endereço
O ensino de custos nas escolas de graduação em enfermagem 
Ivone Maria F. Francisco Valéria Castilho da pesquisadora para o retorno, bem como todos os esclarecimentos para esta devolução.

Nas IES que possuíam mais de uma disciplina relativa à Administração, ou mais de um docente responsável por ministrar aulas teóricas na referida disciplina, foi solicitada a colaboração do Chefe de Departamento ou Coordenador da área, por carta e telefone, para que se houvesse um docente que ministrasse conteúdos relativos a Custos, a este fosse encaminhado o questionário.

O período da coleta de dados estendeuse de outubro de 2001 a março de 2002.

Retornaram 28 questionários, representando $82,35 \%$ da população alvo. A amostra foi composta por 9 (nove) docentes de IES Públicas e 19 docentes de IES Privadas.

\section{Análise dos Dados}

A Análise Descritiva foi realizada sobre as variáveis respostas e as variáveis sócio demográficas dos docentes da Disciplina de Administração Aplicada à Enfermagem. Nesta análise foi adotado, quando possível, distribuições de freqüências e as medidas resumo Média, Desvio Padrão, Mediana, Mínimo e Máximo, Coeficiente de Curtose, Coeficiente de Assimetria e Coeficiente de Variação na produção de estimativas pontuais e Intervalos de Confiança de 95\% na produção de estimativas intervalares. A Análise Inferêncial foi constituída basicamente das análises de associação e de correlação.

\section{APRESENTAÇÃOE DISCUSSÃO DOS RESULTADOS}

\section{Caracterização dos Docentes}

Em relação à variável Idade verificou-se, no global, predomínio da faixa etária 44 a 48 anos com 8 (30,77\%) docentes, valor médio de 44,61(DP=7,86) anos e variação de 34 a 63 anos para a idade.

Quanto ao Tipo de IES da Graduação do Docente, observou-se no global um percentual de 14 (50\%) docentes graduados por Escolas Públicas e 14 (50\%) docentes graduados por Escolas Privadas.

Em relação ao Tempo de Formado a faixa predominante foi de 20 a 24 anos com 10 $(35,71 \%)$ docentes, seguida da faixa de 15 a 19 anos com 7 (25\%) docentes. Observou-se ainda, uma variação de 10 a 39 anos e média de 22,00 (DP=7,51) anos no tempo de formado.

Quanto ao Tempo de Atuação na Graduação, no global, verificou-se que a faixa predominante foi de 2 a 8 anos com 11 (40,74\%) docentes, seguida da faixa de 9 a 15 anos com $8(29,63 \%)$ docentes. A variação observada no tempo de atuação na graduação foi de 2 a 36 anos e a média de $12,41(\mathrm{DP}=8,54)$ anos.

Em relação ao Tempo de Atuação na Pós - Graduação verificou-se, no global, que a faixa predominante foi de 0 a 4 anos com 8 $(66,67 \%)$ docentes, seguida da faixa de 5 a 9 anos com 2 (16,67\%) docentes. A variação observada no tempo de atuação na pós - graduação foi de 0,5 a 23 anos, a média de 4,87 $(\mathrm{DP}=6,51)$ anos e a mediana de 2,5 anos.

Em relação à variável Especializações identificou-se que a Especialização mais freqüente, no global, foi a Administração Hospitalar com 13 (30,23\%) citações, seguida da Enfermagem do Trabalho com 5 (11,62\%) e Enfermagem em Saúde Pública também com 5 $(11,62 \%)$ citações. Uma vez que, o enfoque do estudo foram os docentes da disciplina de Administração, considerando todas as Especializações relacionadas a essa área mencionadas pelos docentes (Administração Hospitalar, Administração de Serviços Públicos de Saúde, Administração em Recursos Humanos, Administração em Enfermagem e Administração e Planejamento em Saúde) tivemos 21 (48,84\%) citações no global.

Em relação à variável Número de Especializações observou-se, no global, que 12 $(42,86 \%)$ docentes cursaram uma especialização, seguido de $8(28,57 \%)$ docentes com duas especializações. Nos limites extremos da tabela obteve-se $3(10,71 \%)$ docentes sem nenhuma especialização, e $5(17,86 \%)$ docentes com três especializações. A variação observada no Número de Especializações foi de 0 a 3 e a média de 1,53 (DP=0,92) especializações.

Em relação à variável Mestrados, observou-se que 22 docentes, no global, possuíam o mestrado, sendo 9 docentes de Escolas Públicas e 13 docentes de Escolas Privadas. O mestrado mais freqüente, no global, foi o Mestrado em Administração com 5 (22,73\%) docentes, seguido do Mestrado em Saúde Coletiva com 3 (13,64\%) e Mestrado em Enfermagem também com $3(13,64 \%)$ docentes. 
Considerando todos os mestrados relacionados à área de Administração (Administração em Serviços de Enfermagem, Administração de Empresas com concentração em Hospitais e Sistemas de Saúde e Administração) obteve-se 9 (40,91\%) citações no global.

Comentando a variável Doutorados, 7 docentes possuíam o Doutorado, sendo 5 de Escolas Públicas e 2 de Escolas Privadas.
Caracterização da Postura do Docente Quanto ao Tema Custos

A Tabela 1 mostra a distribuição da Percepção da Necessidade do Tema Custos na Graduação pelos docentes, segundo o tipo de IES em que trabalhavam e no global.
O ensino de custos nas escolas de graduação em enfermagem

Tabela 1 - Distribuição da Percepção da Necessidade do Tema Custos na Graduação, segundo o tipo de IES e no global (São Paulo, 2002)

\begin{tabular}{|c|c|c|c|c|c|c|}
\hline \multirow{3}{*}{$\begin{array}{c}\text { VARIÁVEL } \\
\text { Percepção da Necessidade do Tema Custos na Graduação }\end{array}$} & \multicolumn{6}{|c|}{ DISTRIBUIÇÃO } \\
\hline & \multicolumn{2}{|c|}{ Privada(19) } & \multicolumn{2}{|c|}{ Pública(9) } & \multicolumn{2}{|c|}{ Global(28) } \\
\hline & $\mathbf{n}$ & $\%$ & $n$ & $\%$ & $\mathbf{n}$ & $\%$ \\
\hline É essencial para subsidiar as argumentações dos enfermeiros & 0 & 0 & 2 & 11,80 & 2 & 4,08 \\
\hline É importante abordar o tema custos & 5 & 15,60 & 2 & 11,80 & 7 & 14,30 \\
\hline Para uma alocação eficaz de recursos & 0 & 0 & 1 & 5,87 & 1 & 2,04 \\
\hline É necessário para a administração de recursos na área da saúde & 6 & 18,80 & 3 & 17,60 & 9 & 18,40 \\
\hline É necessário para o controle de qualidade & 0 & 0 & 1 & 5,87 & 1 & 2,04 \\
\hline É necessário para trabalhar as relações custo benefício & 1 & 3,12 & 2 & 11,80 & 3 & 6,12 \\
\hline É importante para que os enferm. participem da administração dos custos hospitalares & 0 & 0 & 1 & 5,87 & 1 & 2,04 \\
\hline É fundamental para quem trabalha com gerenciamento & 4 & 12,60 & 1 & 5,87 & 5 & 10,20 \\
\hline É importante para a tomada de decisão & 0 & 0 & 1 & 5,87 & 1 & 2,04 \\
\hline Atualmente o controle de custos é uma questão de sobrevivência organizacional & 2 & 6,25 & 2 & 11,80 & 4 & 8,16 \\
\hline É necessário pois o enferm. é um gestor de custos nos div. níveis da org. de saúde & 2 & 6,25 & 0 & 0 & 2 & 4,08 \\
\hline É uma necessidade do mercado de trabalho & 2 & 6,25 & 0 & 0 & 2 & 4,08 \\
\hline É necessário para entender o financiamento do sistema de saúde & 2 & 6,25 & 1 & 5,87 & 3 & 6,12 \\
\hline É imprescindível para o gerenciamento da assistência & 2 & 6,25 & 0 & 0 & 2 & 4,08 \\
\hline É necessário para negociar com os profissionais ligados à saúde & 1 & 3,12 & 0 & 0 & 1 & 2,04 \\
\hline É necessário pois as Org. Saúde convivem com cust. cresc. e recursos limitados & 1 & 3,12 & 0 & 0 & 1 & 2,04 \\
\hline É necessário para uma avaliação econômica dos programas de saúde & 1 & 3,12 & 0 & 0 & 1 & 2,04 \\
\hline É necessário p/ uma avaliação de honorários de enferm. de acordo com o COFEN & 1 & 3,12 & 0 & 0 & 1 & 2,04 \\
\hline É necessário para entender as características da Adm. Pública atual & 1 & 3,12 & 0 & 0 & 1 & 2,04 \\
\hline Não acha importante na graduação e sim na especialização & 1 & 3,12 & 0 & 0 & 1 & 2,04 \\
\hline TOTAL & 32 & 100 & 17 & 100 & 49 & 100 \\
\hline
\end{tabular}

Analisando a Tabela 1 verificou-se que $97,96 \%$ das citações foram favoráveis ao ensino desse tema na graduação, no global obteve-se $9(18,40 \%)$ citações sugerindo que o conhecimento do tema Custos "é necessário para a administração de recursos na área da saúde", seguida de 7 (14,30\%) citações para "é importante abordar o tema Custos" e 5 $(10,20 \%)$ referindo que "é fundamental para quem trabalha com gerenciamento". A importância do tema também foi relacionada às argumentações e negociações dos enfermeiros, à tomada de decisão, à sobrevivência organizacional, à necessidade do mercado de trabalho, etc. Pode-se verificar nas respostas a importância que foi dada ao tema Custos para os enfermeiros que gerenciam Serviços, Unidades e/ou Recursos (humanos, materiais e financeiros) na área da Saúde, e que a introdução, ainda na graduação, deste tema, talvez prepare melhor o aluno para suas futuras responsabilidades.
Em relação à variável Ensino do Tema Custos, identificou-se que, no global, 19 $(67,86 \%)$ docentes ministravam o tema Custos na disciplina de Administração e 9 $(32,14 \%)$ não ministravam esse tema.

Em relação ao nome da Disciplina que Aborda o Tema Custos, predominou, no global, a "Administração Aplicada à Enfermagem" com $10(55,56 \%)$ citações, seguida das disciplinas "Administração em Enfermagem" com $2(11,10 \%)$ e "Administração da Assistência de Enfermagem" também com 2 $(11,10 \%)$ citações.

Em relação à variável Semestre da Disciplina que aborda o Tema Custos verificou-se, no global, $6(31,58 \%)$ citações para o $8^{\circ}$ semestre, seguida de $5(26,32 \%)$ citações para o $7^{\circ}$ semestre. Considerando-se todas as citações incluindo apenas $7^{\circ}$ e $8^{\circ}$ semestres, obteve-se $12(63,16 \%)$ ocorrências. A variação foi do $3^{\circ}$ ao $8^{\circ}$ semestres.

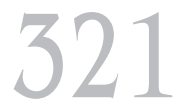

Rev Esc Enferm USP 2004; 38(3):317-25 
Ivone Maria F. Francisco Valéria Castilho
O fato de predominar o ensino deste tema, nos últimos semestres dos Cursos de Graduação, pode vir a indicar um maior preparo dos alunos para sua compreensão, após terem conhecido e estagiado nos diversos setores hospitalares e extra hospitalares.

Em relação à variável Carga Horária Dedicada ao Tema Custos observou-se, no global, que em 12 (70,59\%) citações a carga horária mencionada foi de 4 a 9 horas/aula, seguida de $3(17,65 \%)$ citações com 10 a 15 horas/aula. A variação foi de 4 a 30 horas, a média de 9,12 (DP=7,18) horas/aula e a mediana de 6 horas/aula.

A Tabela 2 mostra a distribuição dos Temas Abordados pelos docentes relaciona- dos à Custos, segundo o tipo de IES em que trabalhavam e no global.

Analisando a Tabela 2, referente aos Temas Abordados na disciplina de Administração relacionados a Custos verificou-se, no global, 17 (21,52\%) citações para “definição e classificação de Custos", seguida de $14(17,72 \%)$ para "sistemas de custeio/ metodologias de apuração de Custos", 13 (16,46\%) para "análise de Custo Benefício", $10(12,66 \%)$ para "orçamento" e $9(11,39 \%)$ para "Políticas Públicas de Financiamento do Setor Saúde". Esses cinco temas totalizaram $79,75 \%$ dos temas relacionados a Custos abordados pelos 19 docentes que ministravam Custos na disciplina de Administração da amostra deste estudo.

Tabela 2 - Distribuição dos Temas Abordados pelos docentes, segundo o tipo de IES e no global (São Paulo, 2002)

\begin{tabular}{|c|c|c|c|c|c|c|}
\hline \multirow{3}{*}{$\frac{\text { VARIÁVEL }}{\text { Temas Abordados }}$} & \multicolumn{6}{|c|}{ DISTRIBUIÇÃO } \\
\hline & \multicolumn{2}{|c|}{ Privada(12) } & \multicolumn{2}{|c|}{ Pública(7) } & \multicolumn{2}{|c|}{ Global(19) } \\
\hline & $\mathbf{n}$ & $\%$ & $\mathbf{n}$ & $\%$ & $\mathbf{n}$ & $\%$ \\
\hline Políticas Públicas de Financiamento do Setor Saúde & 5 & 10,20 & 4 & 13,33 & 9 & 11,39 \\
\hline Definição e Classificação de Custos & 10 & 20,40 & 7 & 23,33 & 17 & 21,52 \\
\hline Sistemas de Custeio/ Metodologias de Apuração de Custos & 7 & 14,30 & 7 & 23,33 & 14 & 17,72 \\
\hline Orçamento & 5 & 10,20 & 5 & 16,68 & 10 & 12,66 \\
\hline Análise de Custo Benefício & 7 & 14,30 & 6 & 20,00 & 13 & 16,46 \\
\hline Custos Hospitalares & 2 & 4,08 & 0 & 0 & 2 & 2,54 \\
\hline Papel do Enfermeiro no Gerenciamento de Custos em Saúde & 2 & 4,08 & 0 & 0 & 2 & 2,54 \\
\hline A importância dos Custos para os Serviços de Enfermagem & 0 & 0 & 1 & 3,33 & 1 & 1,27 \\
\hline Composição da Conta Hospitalar & 2 & 4,08 & 0 & 0 & 2 & 2,54 \\
\hline Princípios Básicos da Contabilidade & 1 & 2,04 & 0 & 0 & 1 & 1,27 \\
\hline Gestão de Custos & 1 & 2,04 & 0 & 0 & 1 & 1,27 \\
\hline Gerenciamento de Custos de Recursos Materiais & 4 & 8,16 & 0 & 0 & 4 & 5,08 \\
\hline Gerenciamento de Custos de Recursos Humanos & 1 & 2,04 & 0 & 0 & 1 & 1,27 \\
\hline Qualidade da Assistência x Custos & 1 & 2,04 & 0 & 0 & 1 & 1,27 \\
\hline Elaboração de Protocolos $x$ Resultados $x$ Custos & 1 & 2,04 & 0 & 0 & 1 & 1,27 \\
\hline TOTAL & 49 & 100 & 30 & 100 & 79 & 100 \\
\hline
\end{tabular}

A Tabela 3 mostra a distribuição do Preparo do Docente para ministrar o tema Cus- tos, segundo o tipo de IES em que trabalhavam e no global.

Tabela 3 - Distribuição do Preparo do Docente para ministrar o tema Custos, segundo o tipo de IES e no global (São Paulo, 2002)

\section{VARIÁVEL} DISTRIBUIÇÃO

\begin{tabular}{|c|c|c|c|c|c|c|}
\hline \multirow[t]{2}{*}{ Preparo do Docente } & \multicolumn{2}{|c|}{ Privada(12 } & \multicolumn{2}{|c|}{ Pública(7) } & \multicolumn{2}{|c|}{ Global(19) } \\
\hline & $\mathbf{n}$ & $\%$ & $\mathbf{n}$ & $\%$ & $\mathbf{n}$ & $\%$ \\
\hline Literatura impressa sobre custos & 8 & 28,57 & 5 & 23,82 & 13 & 26,53 \\
\hline Seminários, palestras, cursos de extensão sobre custos & 8 & 28,57 & 6 & 28,57 & 14 & 28,57 \\
\hline Vivência profissional & 5 & 17,86 & 2 & 9,52 & 7 & 14,29 \\
\hline Disciplinas de Pós-Graduação & 4 & 14,29 & 4 & 19,05 & 8 & 16,33 \\
\hline Pesquisa à Internet & 2 & 7,14 & 1 & 4,76 & 3 & 6,12 \\
\hline Formação anterior ou atual em área correlata & 1 & 3,57 & 2 & 9,52 & 3 & 6,12 \\
\hline Discussão com colegas sobre o tema custos & 0 & 0 & 1 & 4,76 & 1 & 2,04 \\
\hline TOTAL & 28 & 100 & 21 & 100 & 49 & 100 \\
\hline
\end{tabular}


Analisando a Tabela 3, relacionada ao Preparo do Docente para ministrar o tema Custos verificou-se, no global, 14 (28,57\%) citações para "seminários, palestras e cursos de extensão sobre Custos", seguida de 13 (26,53\%) para "literatura impressa sobre Custos", $8(16,33 \%)$ para "disciplinas de pós graduação" e 7 (14,29\%) para "vivência profissional". Essas citações totalizaram 85,72\% das formas de preparo dos docentes. Encontrou-se ainda $3(6,12 \%)$ citações para "pesquisa à Internet" como uma fonte alternativa de acesso à informação.

Pode-se evidenciar a importância da realização de cursos, palestras e seminários sobre o tema, além da divulgação de literaturas específicas sobre Custos para a Enfermagem para auxiliar a capacitação dos docentes.

Sobre a Presença de Dificuldade para ministrar o tema Custos verificou-se, no global, que $14(73,68 \%)$ sentiam dificuldade para ministrar esse tema e $5(26,32 \%)$ não sentiam.

Em relação ao Tipo de Dificuldade para ministrar o tema Custos verificou-se, no global, que $5(35,73 \%)$ docentes citaram a "falta de capacitação específica em relação a Custos" e 4 (28,57\%) docentes citaram sentir dificuldades apenas "em temas avançados sobre Custos", somando 9 (64,30\%) citações. A seguir obteve-se o mesmo número de 1 (7,14\%) citação para: "falta de literatura específica de Custos em Enfermagem",
"Inexistência de modelos de custeio em Enfermagem", "falta de familiaridade com as necessidades empresariais", dificuldade para "mostrar a parte prática/operacional de Custos em campo" e "falta de familiaridade do aluno com o tema".

Em relação à variável Bibliografias utilizadas no ensino de Custos, encontrouse um total de 46 referências indicadas pelos 19 docentes que ministravam esse tema. Verificou-se a maior freqüência, no global, das seguintes Bibliografias: 4 $(5,63 \%)$ citações para "apostilas, artigos e materiais de cursos e palestras", 4 (5,63\%) para o livro "Contabilidade de Custos, inclui ABC" do autor Eliseu Martins, 3 $(4,22 \%)$ para o livro "Gestão de Custos e resultados na Saúde" dos autores Beulke R e Bertó DJ, 3 (4,22\%) para o "Manual de Apuração de Custos Hospitalares" do Ministério da Saúde, 3 (4,22\%) para o livro "Gestão de Custos para Hospitais: conceitos, metodologias e aplicações" do autor Falk JA, 3 (4,22\%) para o livro "Manual de Gerenciamento em Enfermagem" da autora Marx LC, 3 (4,22\%) para a apostila "Sistema de Custos como instrumento de eficiência e qualidade dos Serviços de Saúde" dos autores Medici AC,Marques RM.

A Tabela 4 mostra a distribuição das Sugestões dos docentes para o ensino do tema Custos, segundo o tipo de IES em que trabalhavam e no global.

Tabela 4 - Distribuição das Sugestões dos docentes para o ensino do tema Custos, segundo o tipo de IES e no global (São Paulo, 2002)

\begin{tabular}{|c|c|c|c|c|c|c|}
\hline \multirow{3}{*}{$\begin{array}{l}\text { VARIÁVEL } \\
\text { Sugestões }\end{array}$} & \multicolumn{6}{|c|}{ DISTRIBUIÇÃO } \\
\hline & \multicolumn{2}{|c|}{ Privada(1 } & \multicolumn{2}{|c|}{ Pública(7) } & \multicolumn{2}{|c|}{ Global(19) } \\
\hline & $\mathbf{n}$ & $\%$ & $\mathbf{n}$ & $\%$ & $\mathbf{n}$ & $\%$ \\
\hline Adequar o conteúdo de Custos para graduação & 2 & 20 & 7 & 53,86 & 9 & 39,10 \\
\hline Capacitação do docente para o tema & 0 & 0 & 1 & 7,69 & 1 & 4,35 \\
\hline Formação de grupos de estudo sobre o tema & 0 & 0 & 1 & 7,69 & 1 & 4,35 \\
\hline Estágios em setores do hospital ligados ao tema & 1 & 10 & 1 & 7,69 & 2 & 8,70 \\
\hline Estágios em convênios e Home Care em áreas ligadas ao tema & 0 & 0 & 2 & 15,38 & 2 & 8,70 \\
\hline Destacar o papel do enfermeiro no Gerenciamento de Custos & 1 & 10 & 1 & 7,69 & 2 & 8,70 \\
\hline Apresentar a Unid. de Internação como uma unidade de negócios & 2 & 20 & 0 & 0 & 2 & 8,70 \\
\hline Criar estratégias de ens. que sensibilizem o aluno quanto ao tema & 1 & 10 & 0 & 0 & 1 & 4,35 \\
\hline Criar uma disciplina específica sobre Custos & 2 & 20 & 0 & 0 & 2 & 8,70 \\
\hline Divulgar trabalhos relacionados com o tema & 1 & 10 & 0 & 0 & 1 & 4,35 \\
\hline TOTAL & 10 & 100 & 13 & 100 & 23 & 100 \\
\hline
\end{tabular}

Analisando a Tabela 4, em relação às Sugestões dos docentes para o ensino do tema Custos, obteve-se, no global, $9(39,10 \%)$ citações para a sugestão "adequar o conteú- do de Custos para a graduação", seguida de mesma freqüência de $2(8,70 \%)$ citações para: "estágios em setores do hospital ligados ao tema", "estágios em convênios e Home Care
O ensino de custos nas escolas de graduação em enfermagem 
Ivone Maria F. Francisco Valéria Castilho em áreas ligadas ao tema”, "destacar o papel do enfermeiro no Gerenciamento de Custos", "apresentar a Unidade de Internação como uma unidade de negócios" e "criar uma disciplina específica sobre Custos", somando $82,60 \%$ das sugestões.

Em relação à adequação do conteúdo de Custos para a graduação, os docentes sugeriram: adequar a carga horária, instrumentalizar o aluno para utilização de metodologias de apuração de Custos de Procedimentos de Enfermagem e instrumentalizar o aluno para gerenciar os Custos de Recursos Humanos e Materiais na Enfermagem.

Quanto aos estágios, em setores do Hospital, ligados ao tema Custos, Convênios Médicos e Home Care, os docentes sugeriram os seguintes departamentos: Faturamento, Contabilidade, Setor de Compras e Auditoria de Contas Hospitalares.

Em relação à variável Motivos para não inclusão do tema Custos na disciplina de Administração, verificou-se, no global, 3 $(30 \%)$ citações para a "falta de preparo do docente", $3(30 \%)$ para "sem motivo específico", seguida de $2(20 \%)$ citações para "pequena carga horária da disciplina”.

\section{CORRELAÇÕES E ASSOCIAÇÕES ENTRE VARIÁVEIS DE INTERESSE}

Quanto às correlações e associações entre variáveis de interesse, verificou-se que existe uma correlação estatisticamente significativa entre o Tempo de Formado e Tempo de Atuação na Graduação, Tempo de Atuação na Pós-Graduação e Número de Pós-Graduações indicando que docentes com maior tempo de formado tendem a apresentar maior tempo de atuação na graduação, na pós-graduação e maior número de pós-graduações. Sugere, ainda, que docentes com maior tempo de atuação na graduação tendem a apresentar maior tempo de atuação na pós- graduação. Nota-se, também, uma correlação estatisticamente significativa entre o Número de Pós-Graduações e o tipo de IES em que o docente trabalha, mostrando que docentes com maior número de pós-graduações tendem a trabalhar em Escolas Públicas. Não encontrou-se correlação estatisticamente significativa entre o Número de Pós-Graduações do docente e o ensino do tema Custos.
Verificou-se não haver associação estatisticamente significativa entre o Tipo de IES da Graduação do Docente, o Tipo de IES em que Trabalhava e o Ensino do Tema Custos. Não foi observado, também, associação estatisticamente significativa entre o Tipo de IES em que o Docente Trabalhava, o Ensino do Tema Custos e a presença de Dificuldade para ministrar esse tema.

\section{CONCLUSÕES NO ASPECTO GLOBAL (IES Privadas e Públicas)}

Em relação à caracterização da postura do Docente em relação ao tema Custos, observou-se que $19(67,86 \%)$ docentes, ministravam o tema Custos na graduação; $10(55,56 \%)$ abordavam esse tema na disciplina de "Administração Aplicada à Enfermagem"; 12 (63,16\%) ministravam esse tema nos $7^{\circ}$ e $8^{\circ}$ semestres do curso; 12 (70,59\%) dedicavam de 4 a 9 horas/aula para o ensino de Custos. Quanto ao preparo do docente para ministrar esses temas obteve-se 14 (28,57\%) citações para "seminários, palestras e cursos de extensão sobre Custos" e 13 (26,53\%) citações para "literatura impressa sobre Custos". Identificou-se que $14(73,68 \%)$ docentes sentiam dificuldade para ministrar o tema Custos; 5 (35,73\%) docentes afirmaram que essa dificuldade estava relacionada à "falta de capacitação específica em relação à Custos” e 4 (28,57\%) alegaram dificuldade em "temas avançados sobre Custos". Abordando as sugestões dos docentes para o ensino desse tema encontrou-se 9 $(39,10 \%)$ citações para "adequar o conteúdo de Custos para a Graduação". Em relação aos motivos para não inclusão do tema Custos na disciplina de Administração Aplicada à Enfermagem, obteve-se $3(30 \%)$ citações para "falta de preparo do docente" e $3(30 \%)$ citações para "falta de motivo específico".

Quanto às correlações e associações entre variáveis de interesse verificou-se não haver nenhuma correlação ou associação estatisticamente significativa entre o Ensino do Tema Custos e o Número de Pós-Graduações, o Tipo de IES da Graduação do Docente, e o Tipo de IES em que trabalhava.

\section{CONSIDERAÇÕES FINAIS}

A partir da apresentação e discussão dos resultados, do referencial teórico apresentado, abordando a situação econômica do Se- 
tor Saúde e de diversos autores ${ }^{(1,3-4,7-9)}$ ressaltando a importância do conhecimento de Custos para a Enfermagem, considera-se importante aproximar o aluno de graduação em relação a aspectos econômicos e ao gerenciamento de Custos nos Serviços de Enfermagem, para que esse futuro profissional possa responder aos novos desafios gerenciais com os quais vai se deparar.

O enfermeiro deverá estar apto à atender as demandas das Organizações de Saúde, preocupadas com a elevação crescente de custos, a racionalização de recursos, o controle de desperdícios e a otimização de resultados, assumindo, assim, um papel efetivo na gestão econômica dos Serviços de Enfermagem, tanto no setor Público, como no setor Privado.

Como todo tema relativamente novo, é de se esperar dificuldades, tanto para quem se dispõe a pesquisá-lo, bem como para quem se propõe a lecioná-lo. Para que ocorra a necessária difusão de conhecimentos sobre Custos faz-se necessário, em primeiro lugar capacitar os docentes em relação ao tema, estimulando sua participação em seminários, cursos, especializações ou disciplinas de pós-graduação que permitam o aprofundamento dos seus conhecimentos. Dessa forma, estará o docente preparado para sele- cionar os aspectos mais relevantes sobre Custos, para o aprendizado e formação profissional dos alunos. Fundamental, também, é a intensificação da produção de novos estudos e publicações específicas sobre Custos em Enfermagem. Como sugestão para ampliação da discussão do tema, pode-se apontar a formação de grupos de estudos, incentivo à iniciação científica, estimulo à realização de dissertações e teses sobre esta temática e a promoção de cursos e treinamentos nas Instituições de Saúde.

Igualmente importante é o acesso dos docentes e dos alunos à bibliografia específica nas bibliotecas das Instituições de Ensino Superior, por meio da aquisição de títulos relacionados ao tema, que envolvem as áreas de Enfermagem, Administração Geral, Administração Hospitalar, Contabilidade, Economia e Economia da Saúde.

Considera-se que este trabalho possa contribuir para a Enfermagem, na medida que permitiu conhecer, discutir e divulgar a importância do tema Custos, bem como oferecer aos docentes da disciplina de Administração Aplicada à Enfermagem, ou disciplina equivalente, subsídios que poderão, eventualmente, facilitar a inclusão do tema ou proporcionar o aprofundamento do mesmo.
O ensino de custos nas escolas de graduação em enfermagem

\section{REFERÊNCIAS}

(1) Consejo Internacional de Enfermeras. La calidad, los costos y la enfermeria. Ginebra: CIE; 1993. [Carpeta del Dia Internacional de La Enfermera].

(2) Martins E. Contabilidade de custos. $5^{\mathrm{a}}$ ed. São Paulo: Atlas; 1996.

(3) Marquis BL, Huston CJ. Administração e liderança em enfermagem: teoria e aplicação. $2^{\mathrm{a}}$ ed. Porto Alegre: Artes Médicas; 1999.

(4) Anselmi ML. Quadro de referências para elaboração do orçamento de enfermagem em Instituições Hospitalares. [tese] Ribeirão Preto (SP): Escola de Enfermagem de Ribeirão Preto/USP; 2000.

(5) Medici AC. Financiamento e contenção de custos nas políticas de saúde: tendências atuais e perspectivas futuras. Planej Polít Públicas 1990; (4):83-93.
(6) Medici AC, Marques RM. Sistema de custos como instrumento de eficiência e qualidade dos Serviços de Saúde. Cad Fundap 1996; 16(19):47-59.

(7) Aburdene P, Naisbitt J. Megatendências para as mulheres. Rio de Janeiro: Rosa dos Tempos; 1993.

(8) Anselmi ML, Nakao JRS. A Enfermagem no processo de gestão econômica econômica dos serviços de saúde: limites e possibilidades. Rev Bras Enferm 1999; 52(2):223-32.

(9) Almeida MH. Custos hospitalares na enfermagem. Rio de Janeiro: Cultura Médica; 1984. 